\title{
Improving Separation Assurance Stability through Trajectory Flexibility Preservation
}

\author{
Husni R. Idris ${ }^{*}$ and Ni Shen ${ }^{\dagger}$ \\ Engility Corporation, Billerica, MA, 01821 \\ David J. Wing \\ NASA Langley Research Center, Hampton, VA, 23681
}

\begin{abstract}
New information and automation technologies are enabling the distribution of tasks and decisions from the service providers to the users of the air traffic system, with potential capacity and cost benefits. This distribution of tasks and decisions raises the concern that independent user actions will decrease the predictability and increase the complexity of the traffic system, hence inhibiting and possibly reversing any potential benefits. One such concern is the adverse impact of uncoordinated actions by individual aircraft on the stability of separation assurance. For example, individual aircraft performing self-separation may resolve predicted losses of separation or conflicts with some traffic, only to result in secondary conflicts with other traffic or with the same traffic later in time. In answer to this concern, this paper proposes metrics for preserving user trajectory flexibility to be used in self-separation along with other objectives. The hypothesis is that preserving trajectory flexibility will naturally reduce the creation of secondary conflicts by bringing about implicit coordination between aircraft. The impact of using these metrics on improving selfseparation stability is investigated by measuring the impact on secondary conflicts. The scenarios analyzed include aircraft in en route airspace with each aircraft meeting a required time of arrival in a twenty minute time horizon while maintaining separation from the surrounding traffic and using trajectory flexibility metrics to mitigate the risk of secondary conflicts. Preliminary experiments showed promising results in that the trajectory flexibility preservation reduced the potential for secondary conflicts.
\end{abstract}

\section{Nomenclature}

\author{
ADP Adaptability \\ RBT Robustness \\ RTA Required Time of Arrival \\ $(\mathrm{t}, \mathrm{x}, \mathrm{y}) \quad$ (time, $\mathrm{x}$-location, $\mathrm{y}$-location) \\ V Ground speed \\ $\mathrm{h}_{\mathrm{i}}, \mathrm{h}_{\min }, \mathrm{h}_{\max } \quad$ Heading with its maximum and minimum values \\ Traj Trajectory \\ $\mathrm{P}_{\mathrm{i}} \quad$ Probability of trajectory instance traj $\mathrm{j}_{\mathrm{i}}$ \\ * Principal Research Engineer, 300 Concord Road, Suite 400, AIAA Member \\ ${ }^{\dagger}$ Research Analyst, 300 Concord Road, Suite 400, AIAA Member \\ * Principal ATM Research Engineer, NASA LaRC, Mail Stop 152, AIAA Member
}




$\begin{array}{ll}\mathrm{P}_{\mathrm{c}} & \text { Probability of constraint situation } \mathrm{c} \\ \mathrm{P}_{\mathrm{f}} & \text { Probability of feasibility } \\ \mathrm{P}_{\mathrm{f}, \mathrm{c}} & \text { Probability of feasibility in constraint situation } \mathrm{c} \\ \mathrm{f}(\mathrm{t}, \mathrm{x}, \mathrm{y}) & \text { Number of feasible trajectories from }(\mathrm{t}, \mathrm{x}, \mathrm{y}) \text { to destination } \\ \mathrm{f}_{\mathrm{c}}(\mathrm{t}, \mathrm{x}, \mathrm{y}) & \text { Number of feasible trajectories from }(\mathrm{t}, \mathrm{x}, \mathrm{y}) \text { to destination in situation } \mathrm{c} \\ \mathrm{i}(\mathrm{t}, \mathrm{x}, \mathrm{y}) & \text { Number of infeasible trajectories from }(\mathrm{t}, \mathrm{x}, \mathrm{y}) \text { to destination } \\ \mathrm{i}_{\mathrm{c}}(\mathrm{t}, \mathrm{x}, \mathrm{y}) & \text { Number of infeasible trajectories from }(\mathrm{t}, \mathrm{x}, \mathrm{y}) \text { to destination in situation } \mathrm{c} \\ \mathrm{N}(\mathrm{t}, \mathrm{x}, \mathrm{y}) & \text { Number of all trajectories from }(\mathrm{t}, \mathrm{x}, \mathrm{y}) \text { to destination } \\ \mathrm{g}(\mathrm{x}, \mathrm{y}) \text { or } \mathrm{g}_{\mathrm{k}}(\mathrm{x}, \mathrm{y}) & \text { Number of trajectories from } \mathrm{k}=(\mathrm{t}, \mathrm{x}, \mathrm{y}) \text { to next time step } \\ \varepsilon & \text { Duration between time increments } \\ \Delta \mathrm{T} & \text { Time horizon for conflict detection }\end{array}$

\section{Introduction}

$\mathrm{I}$ order to handle the expected increase in air traffic, the Next Generation Air Transportation System (NextGen) will introduce key transformations in Air Traffic Management (ATM). ${ }^{1}$ Three examples are: increasing information sharing through net-enabled information access; making access to National Airspace System (NAS) resources dependent on aircraft equipage; and aircraft trajectory-based operations enabled by aircraft ability to precisely follow customized four dimensional (4D) trajectories. ${ }^{1}$ These capabilities enable shifting the ATM system towards a distributed architecture. ${ }^{2}$ For example, NextGen is investigating delegating more responsibility for traffic separation to the $\operatorname{pilot}^{2,3}$ and delegating more responsibility to airline operation centers for traffic flow management ${ }^{3,4}$. Enabling the gains of distributed decision making depends on the ability of distributed actions, by airborne and ground-based agents, to maintain safety and efficiency at acceptable levels.

Research on distributed ATM has focused, in part, on the distribution of separation responsibility between pilots and controllers, where pilots are delegated increased responsibility for separation assurance relative to current centralized operations. ${ }^{5,6,7,9}$ One concern in distributed operations is the adverse impact of uncoordinated actions by individual aircraft on the stability of separation assurance. For example, individual aircraft performing selfseparation may resolve predicted losses of separation or conflicts with some traffic, only to result in secondary conflicts with other traffic or with the same traffic later in time. In answer to this concern, this paper proposes metrics for preserving user trajectory flexibility to be used in self-separation along with other objectives. Trajectory flexibility preservation enables an aircraft to plan its trajectory such that it preserves a requisite level of maneuvering flexibility in order to accommodate later disturbances caused, for example, by other traffic and weather activity. As discussed in Idris et al., although flexibility preservation does not explicitly coordinate between aircraft, it assists each by reducing the risk of conflict due to the potential behavior of the surrounding traffic, thus resulting in implicit coordination. ${ }^{10,11}$ The hypothesis is that preserving trajectory flexibility will make user actions naturally reduce the creation of secondary conflicts by bringing about implicit coordination between aircraft.

Flexibility preservation complements separation assurance both within the conflict resolution horizon and beyond it to an extended flexibility planning horizon. Beyond the conflict resolution horizon, flexibility preservation plans the aircraft trajectory to minimize its exposure to disturbances such as weather cells and dense traffic. In previous efforts, the authors showed that using trajectory flexibility in a long time horizon mitigated traffic complexity by inducing self organization in the traffic situation as well as reducing measures of traffic complexity such as dynamic density. ${ }^{12,13,14,15}$ Within the conflict resolution horizon, which is the focus of this paper, flexibility aids in selecting from many conflict resolution solutions one that afford the aircraft more flexibility, for example, to adapt to potential subsequent intruder traffic behavior. One example is shown in Figure 1, where, in the left side of the figure, two conflicts are predicted within the look-ahead horizon between two unrelated pairs of aircraft. If the two aircraft labeled "ownship" maneuvered to resolve their respective conflicts, without coordination, a new coincidental conflict may arise between them as shown by the dotted lines. Although flexibility preservation does not explicitly coordinate between the two ownship aircraft, it assists each one in reducing the risk of secondary conflict due to the potential behavior of the surrounding traffic, thus resulting in a form of implicit coordination. 
Hence with this function, each ownship aircraft may select a more flexible trajectory anticipating the potential maneuver of the other aircraft and maneuvering away from it as shown in the right side of Figure 1.
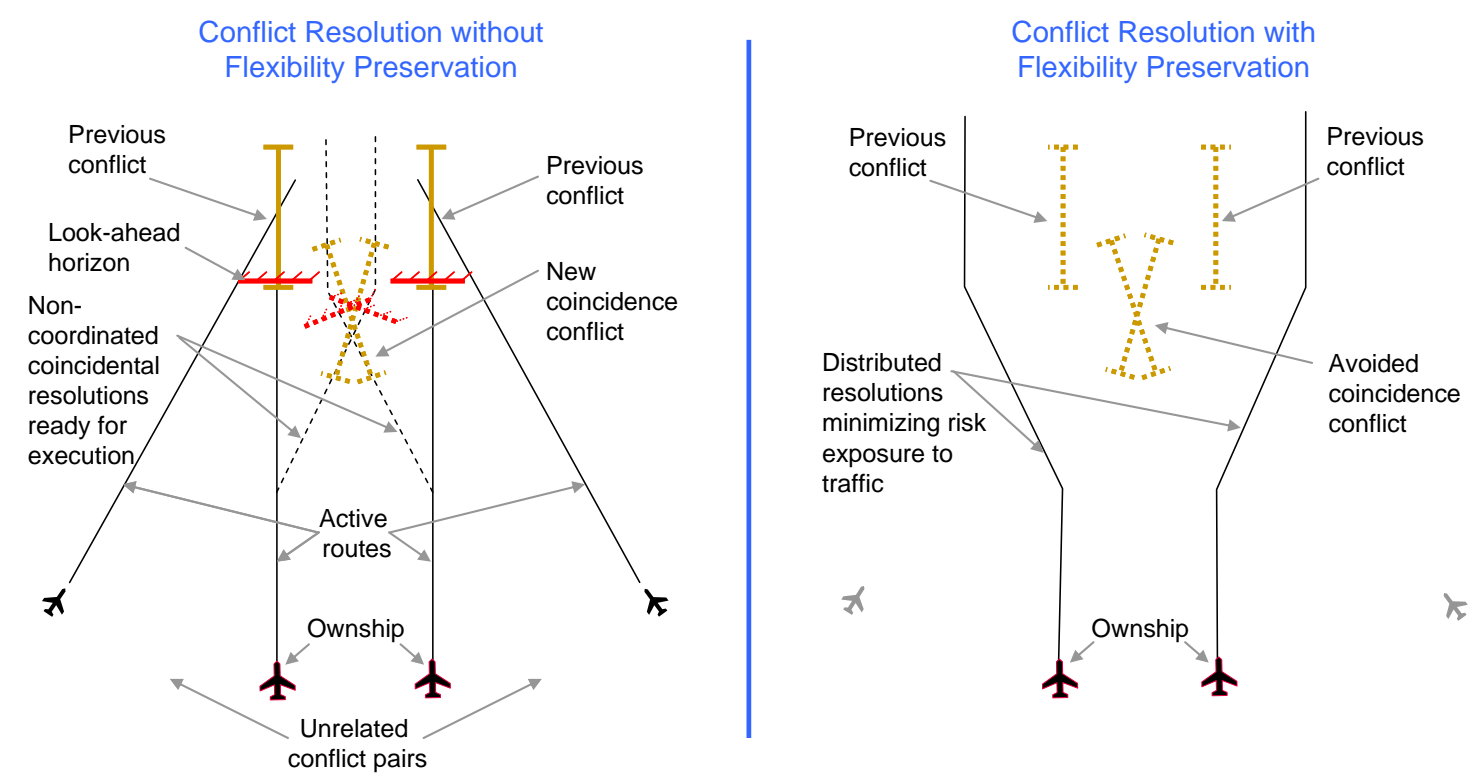

Figure 1 Trajectory flexibility preservation avoiding a coincidence conflict

Trajectory flexibility metrics have been defined in previous work to represent robustness and adaptability to the risk of violating separation, airspace hazards, and traffic flow management constraints. ${ }^{11-13}$ In this paper, the impact of trajectory planning that preserves these metrics on the stability of separation assurance is analyzed. The impact is measured using the rate of secondary conflicts resulting from uncoordinated separation assurance actions and hence the need to update the trajectory. A scenario is analyzed in two-dimensional en route airspace, where each aircraft must meet a required time of arrival (RTA) in a twenty minute time horizon using speed and heading degrees of freedom. Simultaneously, each aircraft preserves its trajectory flexibility, using the defined metrics, to mitigate the risk of loss of separation with intruder aircraft. The scenario used for analysis depicts the hypothetical situation shown in Fig. 1 using an implementation in MATLAB. In order to create the coincidence situation of Fig. 1, each aircraft is made aware of the intruder aircraft existence and original trajectories, but not their intended maneuvers for meeting the RTA or conflict resolution. The experiments showed promising results in terms of reducing the potential of secondary conflicts when using trajectory flexibility relative to using the shortest path in the selection of solutions. The metrics are described in the next section, followed by a description of the method used for metric estimation and trajectory generation. Then the analysis scenario and observations are described, ending with concluding remarks.

\section{Metrics}

Metrics that represent trajectory flexibility have been developed and reported in previous papers. ${ }^{12,13}$ They are summarized briefly in this section. To test the impact of preserving trajectory flexibility on separation assurance stability, metrics measuring the stability performance of self-separation through the creation of secondary conflicts are also briefly described in this section.

\section{A. Trajectory Flexibility Metrics}

The notion of "trajectory flexibility" was defined in Idris et al. ${ }^{11}$ as the ability of the trajectory (and hence the aircraft following the trajectory) to abide by all constraints imposed on it while mitigating its exposure to risks that cause violation of these constraints. The constraints intend to achieve ATM and aircraft objectives and include speed and heading limits, RTAs, and separation minima. They define the trajectory solution space. Risk of constraint violation is represented by disturbances that cause the aircraft trajectory to violate or potentially violate constraints. Disturbances were classified in Idris et al. ${ }^{11-13}$ into state disturbances that result in aircraft state deviation along its trajectory or constraint disturbances such as new constraints or modifications of currently imposed or known potential constraints. 
Two trajectory characteristics relevant to measuring this notion of flexibility have been identified: robustness and adaptability. ${ }^{11}$ Metrics have been proposed for robustness and adaptability based on estimating the number of feasible trajectories available to the aircraft to accommodate disturbances. ${ }^{12,13}$ They are summarized here briefly. In order to support these definitions and estimation methods, the aircraft is assumed to follow segments of discrete time length, where instantaneous heading and speed changes can only occur at discrete instances in time that are $\varepsilon$ apart. Also, heading $h$ and speed $V$ take discrete values between $h_{\min }$ and $h_{\max }$ and between $V_{\text {min }}$ and $V_{\max }$ and are constant along each segment. (Altitude is not considered in this paper.) In addition to simplifying the estimation method, these assumptions are reasonable from an operational point of view considering the intended application of the trajectory flexibility metrics. Namely, the metrics are intended for relative comparison of trajectories over a long time horizon suitable for strategic planning (typical of traffic flow management and strategic conflict resolution planning horizons) as opposed to tactical maneuvering (where the dynamics of the speed and heading change are relevant).

(1) Robustness is defined as the ability of the aircraft to keep its planned trajectory unchanged in response to the occurrence of disturbances, for example, no matter which trajectory or conflict instances materialize. A robustness metric RBT(t, $x, y)$ is measured with the probability of feasibility $\mathrm{P}_{\mathrm{f}}($ traj) of the trajectory (traj) starting from a state $(t, x, y)$ and ending at another state such as (RTA, $\left.x_{\text {dest }}, y_{\text {dest }}\right)$. $P_{f}($ traj $)$ can be estimated with partial information about state and constraint disturbances, modeled with probability distributions that represent the risk of constraint violation. As an example, the constraints are modeled with constraint situations $\mathrm{c}$ of quantity $\mathrm{C}$, each with a probability $P_{c}$ with $\sum_{c=1: C} P_{c}=1$. Each constraint situation $c$ divides the total set of trajectories $N(t, x, y)$ into two mutually exclusive subsets: $f_{c}(t, x, y)$ the set of feasible trajectories and $i_{c}(t, x, y)$ the set of infeasible trajectories, both with respect to $c$. Then, the following formula can be derived for robustness RBT(t, $x, y)$; see Idris et al. ${ }^{12,13}$ for more details:

$$
\begin{aligned}
& P_{f, c}(t, x, y)=\sum_{\text {set of feasible trajectory instancesi }} P\left(\operatorname{traj}_{i}\right)=\frac{f_{c}(t, x, y)}{N(t, x, y)}=\frac{f_{c}(t, x, y)}{f_{c}(t, x, y)+i_{c}(t, x, y)}, \\
& R B T(t, x, y)=P_{f}(t, x, y)=\sum_{c=1: C} P_{c} \times P_{f, c}(t, x, y)=\sum_{c=1: C} P_{c} \times \frac{f_{c}(t, x, y)}{f_{c}(t, x, y)+i_{c}(t, x, y)}
\end{aligned}
$$

where $\mathrm{P}_{\mathrm{f}, \mathrm{c}}$ is the probability of feasibility of the trajectory traj in a constraint situation $\mathrm{c}$, which is the ratio of the number of feasible trajectories $\mathrm{f}_{\mathrm{c}}$ to the total number of trajectories $\mathrm{N}$, if the probability $\mathrm{P}\left(\mathrm{traj}_{\mathrm{i}}\right)$ of each trajectory instance traj $\mathrm{j}_{\mathrm{i}}$, is equal to $1 / \mathrm{N}(\mathrm{t}, \mathrm{x}, \mathrm{y})$.

(2) Adaptability is defined as the ability of the aircraft to change its planned trajectory in response to the occurrence of a disturbance that renders the current planned trajectory infeasible. An adaptability metric ADP(t, $x$, $y$ ) is measured by the number of feasible trajectories $f(t, x, y)$ (with respect to all constraints) that are available for the aircraft to use at $(t, x, y)$ to regain feasibility. Then, given the probability distribution $\left(\mathrm{P}_{c}\right)$ of each constraint situation $\mathrm{c}$ of $\mathrm{C}, \mathrm{ADP}$ may be estimated by the average over $\mathrm{C}$ :

$$
\operatorname{ADP}(t, x, y)=f(t, x, y)=\sum_{c=l: C} P_{c} \times f_{c}(t, x, y)
$$

Adaptability decreases as the aircraft moves along a trajectory because the number of feasible trajectories decreases. The special case of robustness given by (1) (assuming totally random state disturbances) increases over time because as the number of feasible trajectories (numerator) decreases the total number of trajectories (denominator) decreases more rapidly by both infeasible and feasible trajectories.

\section{B. Separation Assurance Stability Metrics}

The impact of planning trajectories using the adaptability and robustness metrics on the stability of separation assurance was assessed using the number of secondary conflicts as an inverse measure of effectiveness. A secondary conflict results when aircraft, in their attempt to resolve primary conflicts, produce new conflicts between the same aircraft pair due to the lack of coordination. Secondary conflicts indicate the stability of separation assurance in terms of the need to re-plan the trajectories to resolve the secondary conflicts. The number of secondary conflicts at an instant of time is measured as the number of aircraft pairs with impending conflict in a time horizon $\Delta \mathrm{T}$. Trajectory-based conflicts in a ten-minute conflict prediction time horizon are detected in this analysis. For each time step, an aircraft pair with horizontal separation less than the minimal horizontal separation requirement (a five nautical mile separation requirement was used) in the next ten minutes is counted as an impending conflict. 


\section{Trajectory Generation Algorithm}

A dynamic programming algorithm was used to generate an aircraft trajectory using the robustness and adaptability metrics. Because the intention of this analysis is to test a hypothesis rather than a real-time application, the dynamic programming approach was selected due to its simple formulation and despite its numerical and storage limitations. First, the trajectory solution space is built as a tree of discrete states connected according to reachability by the allowable discrete speed and heading values over the discrete time increments. Second, the robustness and adaptability metrics are estimated at each state. Third and finally, a back-propagation algorithm computes a cost function and builds a trajectory that optimizes the cost function.

\section{Flexibility Metric Estimation}

Under the assumptions of discrete time and degrees of freedom, the number of trajectories was estimated using a convolution and filtering technique. ${ }^{11}$ Fig. 2 demonstrates this method for calculating $\mathrm{f}_{\mathrm{c}}(\mathrm{t}, \mathrm{x}, \mathrm{y})$ from any point $(\mathrm{t}, \mathrm{x}$, $y)$ to a destination specified by a point $\left(\mathrm{RTA}, \mathrm{x}_{\text {dest }}, \mathrm{y}_{\text {dest }}\right)$ and a tolerance circle around it in the $\mathrm{x}-\mathrm{y}$ plane, in a constraint situation $\mathrm{c}$ that includes an instance of a potential conflict. The three-dimensional space is discretized into time steps $\varepsilon$ apart, where in each time step, the $\mathrm{x}$-y plane is discretized into square cells. The function $\mathrm{f}_{\mathrm{c}}(\mathrm{t}, \mathrm{x}, \mathrm{y})$ is estimated for each cell. Assume the function $f_{c}\left(t_{j}, x, y\right)$ at time $t_{j}$ is known. The function $f_{c}\left(t_{j-1}, x, y\right)$ at the previous time step $t_{j-1}$ can be obtained by convoluting $f_{c}\left(t_{j}, x, y\right)$ and the function $g_{k}(x, y)$, which represents the number of feasible trajectories that reach from a point $\mathrm{k}=\left(\mathrm{t}_{\mathrm{j}-1}, \mathrm{x}(\mathrm{k}), \mathrm{y}(\mathrm{k})\right)$ at time step $\mathrm{t}_{\mathrm{j}-1}$ to the next time step $\mathrm{t}_{\mathrm{j}}$. The function $\mathrm{g}_{\mathrm{k}}(\mathrm{x}, \mathrm{y})$ is shown as a conical shell in Fig. 2. However, if the point $\mathrm{k}$ is infeasible (for example due to loss of separation) then $f_{c}\left(t_{j-1}, x(k), y(k)\right)=0$. This requires a filtering step before each convolution operation to zero out the values at infeasible states. Substituting a dummy variable $\tau$ to denote sliding the point $\mathrm{k}$ in the $\mathrm{x}-\mathrm{y}$ plane, the function $\mathrm{f}_{\mathrm{c}}\left(\mathrm{t}_{\mathrm{j}-1}, \mathrm{x}, \mathrm{y}\right)$ is given by the following equation, representing convolution and filtering for infeasibility:

$$
\begin{array}{ll}
\mathrm{f}_{\mathrm{c}}\left(\mathrm{t}_{\mathrm{j}-1}, \mathrm{x}, \mathrm{y}\right)=\sum_{\lambda} \sum_{\tau} \mathrm{f}_{\mathrm{c}}\left(\mathrm{t}_{\mathrm{j}}, \tau, \lambda\right) \mathrm{g}(\mathrm{x}-\tau, \mathrm{y}-\lambda) & \text { if feasible } \\
\mathrm{f}_{\mathrm{c}}\left(\mathrm{t}_{\mathrm{j}-1}, \mathrm{x}, \mathrm{y}\right)=0 & \text { if infeasible. }
\end{array}
$$

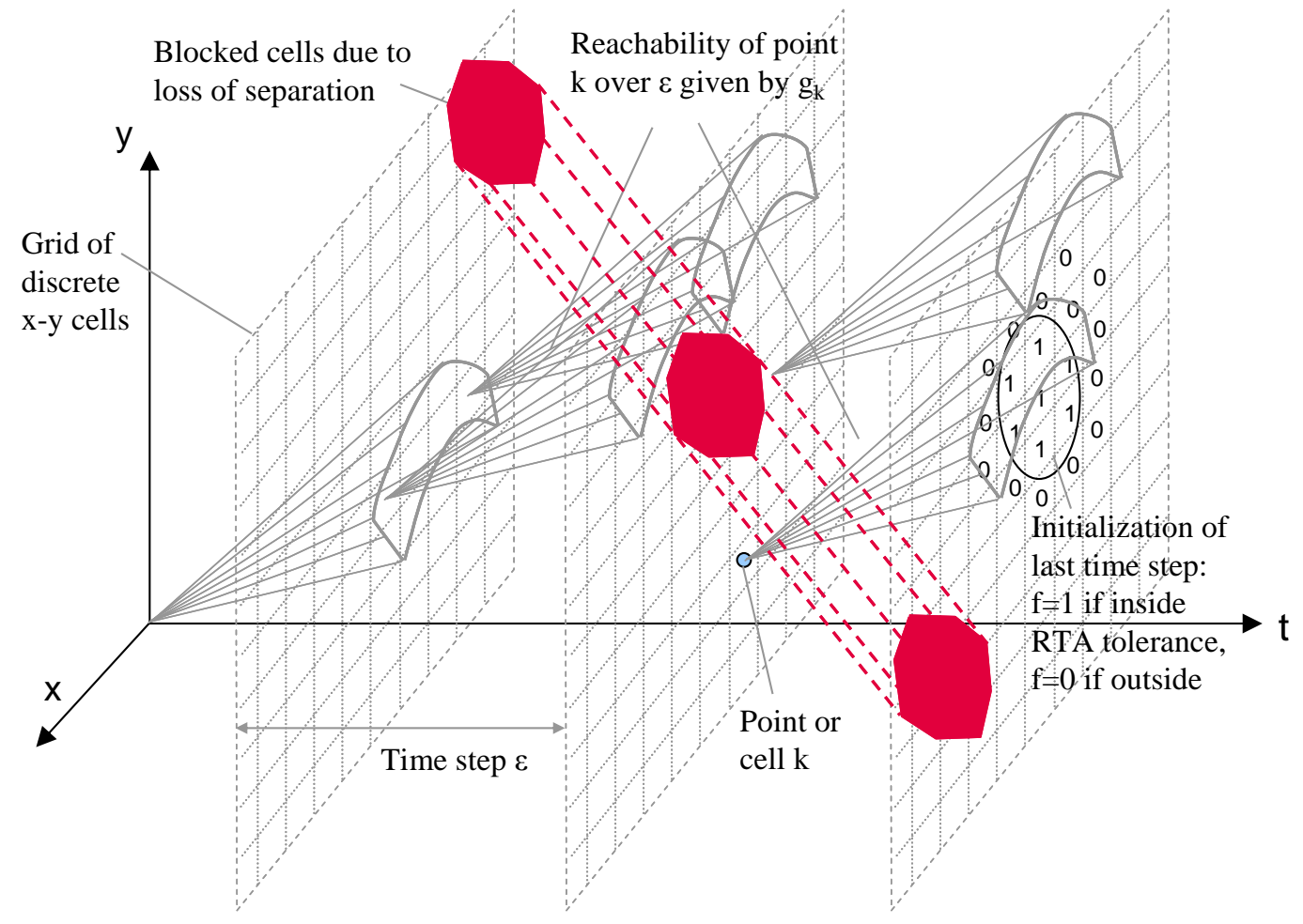

Figure 2. Discrete estimation of number of feasible trajectories 
This operation is applied starting from the destination step $\mathrm{t}=\mathrm{RTA}$ and proceeding backwards to the current state. The destination time step is initialized by setting $\mathrm{f}_{\mathrm{c}}(\mathrm{RTA}, \mathrm{x}, \mathrm{y})=1$ at the feasible states and zero elsewhere, as shown in Fig. 2. To compute the total number of trajectories, $\mathrm{N}(\mathrm{t}, \mathrm{x}, \mathrm{y})$ used in the denominator of the robustness metric RBT, certain constraints are excluded from the filtering process (namely the constraints with respect to which robustness is computed). In this paper, robustness only to loss of separation with traffic is considered. Therefore, the numerator filtering was applied to all cells that led to separation loss as well as cells that led to violating speed and heading limits or violating the RTA constraint. On the other hand, filtering ignored loss of separation but was applied to the RTA and heading and speed limit constraints for calculating the denominator.

Separation zones were modeled as circles with given radii surrounding each intruder aircraft trajectory. Because a trajectory consists of discrete segments, each with constant speed and heading, the circle moves with constant speed and heading for the duration of each segment. In each segment, the circle is enclosed with eight tangent planes, each two opposing tangents resulting from a combination of heading and speed limits of the ownship aircraft relative to the intruder (there are four such combinations). A cell loses (or is imminent to lose) separation if it falls on the inside of all eight planes, within the time duration of the segment. A trajectory segment between two cells (part of the reachabilityfunction g) is considered infeasible if it intersects the polygon around the intruder aircraft trajectory. Hence, a cell is considered infeasible also if all the trajectory segments connecting the cells in the previous time step to it intersect the polygon around the intruder aircraft trajectory. Under probabilistic models of disturbances, the estimation process is repeated for each constraint situation $c$. Then, the estimates $f_{c}(t, x, y)$ are averaged over all situations $C$ to obtain the adaptability or robustness metrics (1) and (2). Examples of ADP and RBT are shown in Fig. 3 and 4 for one of the analysis cases.

\section{Cost Function and Trajectory Building}

Using recursive back-propagation, starting from the final time step, the minimum cost of proceeding from each cell to the destination is computed and stored. This minimum cost $\mathrm{Q}(\mathrm{t}, \mathrm{x}(\mathrm{k}), \mathrm{y}(\mathrm{k}))$ for each cell $\mathrm{k}$ is computed by minimizing, over its reachable cells given by $\left\{(\mathrm{t}+1, \mathrm{x}, \mathrm{y}): \mathrm{g}_{\mathrm{k}}(\mathrm{t}+1, \mathrm{x}, \mathrm{y})=1\right\}$ in the next time step $\mathrm{t}+1$, the sum of the minimum cost $\mathrm{Q}(\mathrm{t}+1, \mathrm{x}, \mathrm{y})$ already computed for each of the reachable cells $(\mathrm{t}+1, \mathrm{x}, \mathrm{y})$ plus the cost of proceeding from $\mathrm{k}$ to that cell, given for short by $\mathrm{q}(\mathrm{k} \rightarrow(\mathrm{t}+1, \mathrm{x}, \mathrm{y}))$. A generic formula is:

$$
\mathrm{Q}(\mathrm{t}, \mathrm{x}(\mathrm{k}), \mathrm{y}(\mathrm{k}))=\operatorname{Min}_{\mathrm{x}, \mathrm{y}: \mathrm{g}_{\mathrm{k}}(\mathrm{x}, \mathrm{y})>1}\{\mathrm{Q}(\mathrm{t}+1, \mathrm{x}, \mathrm{y})+\mathrm{q}(\mathrm{k} \rightarrow(\mathrm{t}+1, \mathrm{x}, \mathrm{y})\}
$$

After storing the optimal costs for each cell, a forward loop builds a trajectory by tracing the optimal cells starting from the initial state. Any ties are broken randomly. Three functions for the local cost $q$ were used in the experiments reported in this paper. The first equation (4) represents a shortest path solution selection where path length is minimized. This shortest path solution is used as a baseline for comparison. Then, equation 5 and 6 represent maximizing adaptability and maximizing robustness, respectively:

$$
\begin{aligned}
& \mathrm{q}(\mathrm{k} \rightarrow(\mathrm{t}+1, \mathrm{x}, \mathrm{y}))=\operatorname{distance}(\mathrm{k} \rightarrow(\mathrm{t}+1, \mathrm{x}, \mathrm{y}))=\text { dist } \\
& \mathrm{q}(\mathrm{k} \rightarrow(\mathrm{t}+1, \mathrm{x}, \mathrm{y}))=-\operatorname{ADP}(\mathrm{k}) \\
& \mathrm{q}(\mathrm{k} \rightarrow(\mathrm{t}+1, \mathrm{x}, \mathrm{y}))=-\mathrm{RBT}(\mathrm{k})
\end{aligned}
$$

Fig. 3 shows an example of the adaptability metric (ADP), at one time step of the solution space, for an aircraft that encounters a line of three hazards with holes in between. Color shades are used to depict the log of the number of feasible trajectories. Fig. 4 shows an example of the robustness metric (RBT), at one time step of the solution space, using color shades. The number of trajectories is zero in the polygons surrounding the hazards, as indicated by the darkest color. Note that adaptability is highest near the center of the solution space around the central hazard, while robustness is highest near the extremities of the solution space away from the central hazard. Also note that robustness here is with respect to the hazards and loss of separation only and not to the RTA constraint or the speed and heading limits. Finally it should be noted that the solution space is smaller in Fig. 4 because it is an earlier time step and that these figures are in a relative frame with respect to the aircraft (hence the hazard y-location is 120 nautical miles rather than zero). 


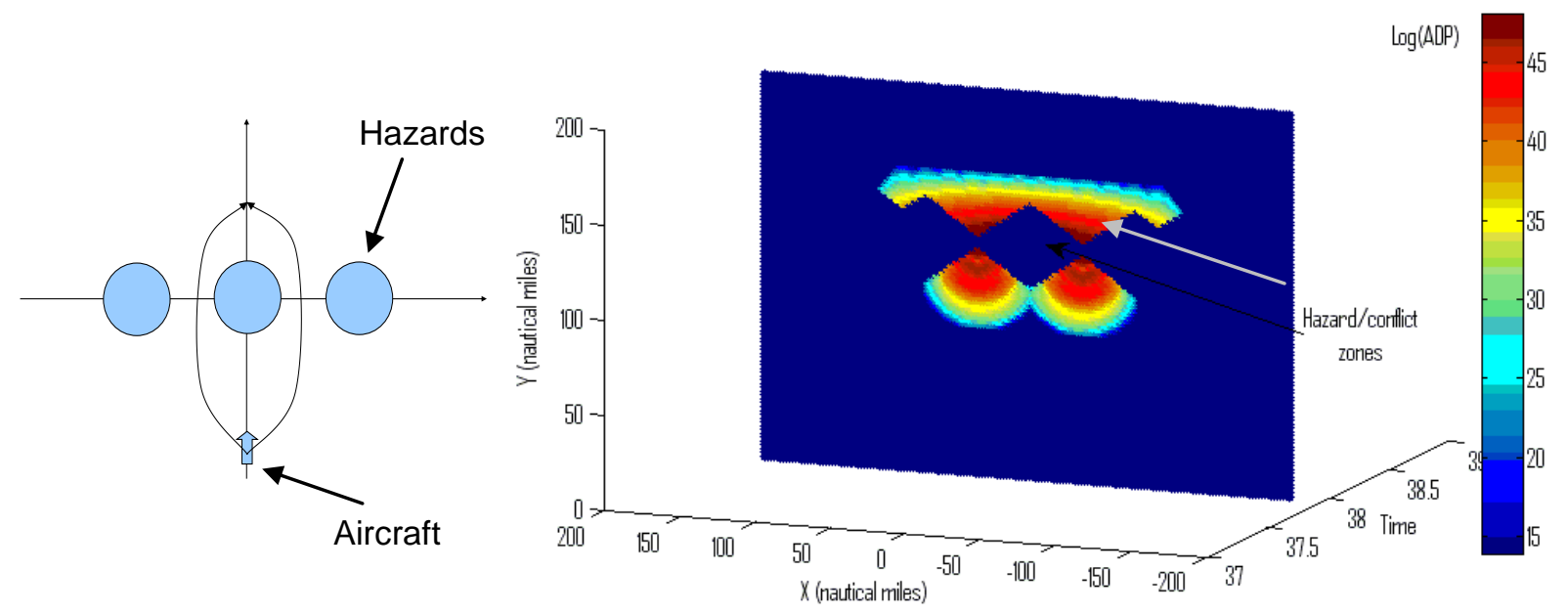

Figure 3. Example of adaptability metric map
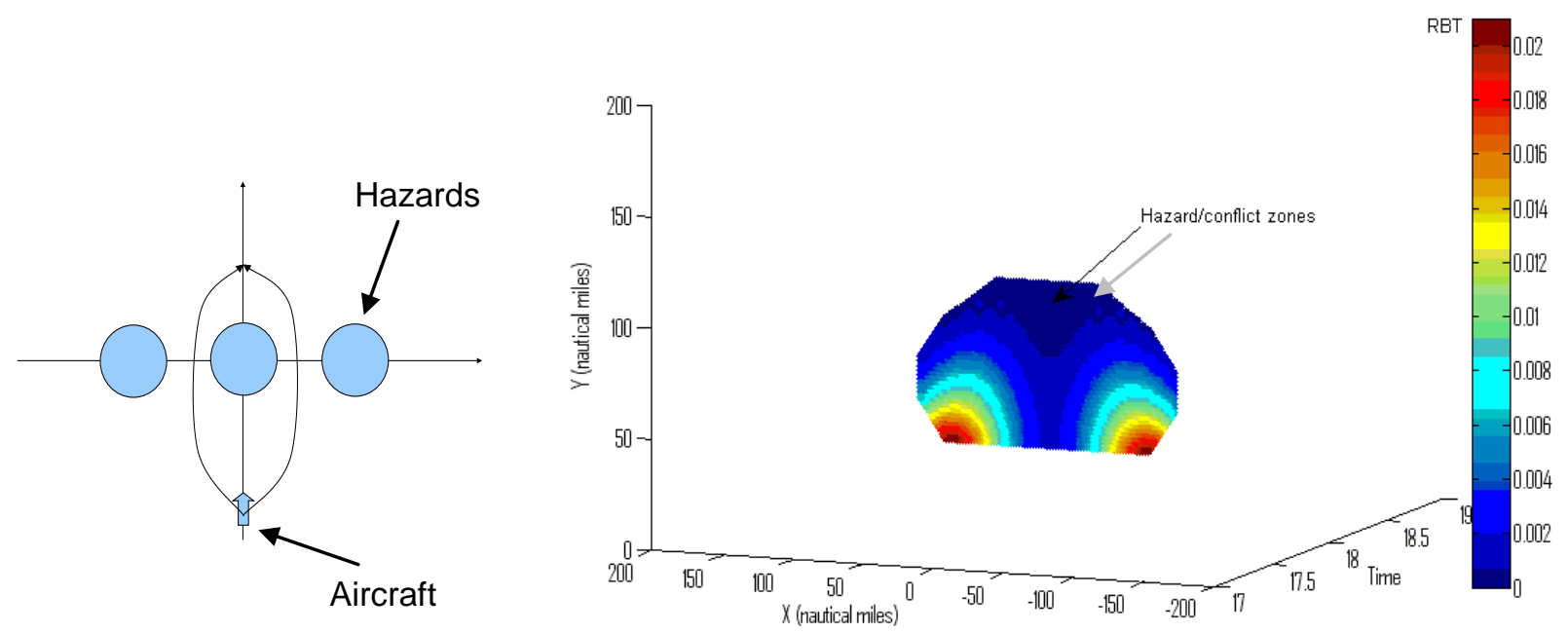

Figure 4. Example of robustness metric map

\section{Self-separation Stability Impact Analysis}

The estimation technique and trajectory optimization algorithm were implemented in a MATLAB tool. The resulting trajectories were analyzed using the separation loss metrics described in Section III.B. First, the scenarios analyzed in this paper are described. Then, observations are made on the impact of trajectory planning, using the three cost functions (4) through (6).

\section{E. Analysis Scenario}

The scenario used for analysis depicts a variation on the hypothetical situation that was shown in Fig. 1. An implementation in MATLAB is demonstrated in Fig. 5. The scenario consists of six aircraft, aircraft 1-4 having to meet an RTA at a 100 nautical mile distance along their line of sight, and aircraft 5 and 6 having to meet an RTA at 107 nautical mile distance along their line of sight at 60 degrees and 120 degrees, respectively. Aircraft 1 and 3 head on each other, and aircraft 2 and 4 head on each other. The routes of aircraft 1 and 3 are 20 nautical miles apart from those of aircraft 2 and 4, horizontally. In this scenario, two aircraft (i.e., 5 and 3) pose a risk of separation loss with aircraft 1. Symmetrically, two aircraft (i.e., 6 and 4) pose a risk of separation loss with aircraft 2. Aircraft 5 and 6 pose a risk of separation loss with each other. All of the aircraft maneuver simultaneously to meet their respective RTA and mitigate the risk of separation loss. In order to create the coincidence situation of Fig. 1, each aircraft is 
made aware of all the other five aircrafts' original trajectories, but it has no information about how they will maneuver. All the aircraft maneuver by selecting a speed and path stretch profile, with a speed range between 260 and 340 knots with 10 knot increments and a heading range between plus and minus 60 degrees relative to their line of sight with 10 degree increments. Time increments of 2 minutes and square $x-y$ cells of 2 nautical miles are used in the estimation of the number of trajectories for the adaptability and robustness metrics. The original trajectories are assumed along a straight line to the destination and with a speed of 300 knots. The original trajectories of the intruder aircraft are surrounded by separation zones (with a 5-mile radius) that are avoided by each ownship aircraft.

(a) Shortest path

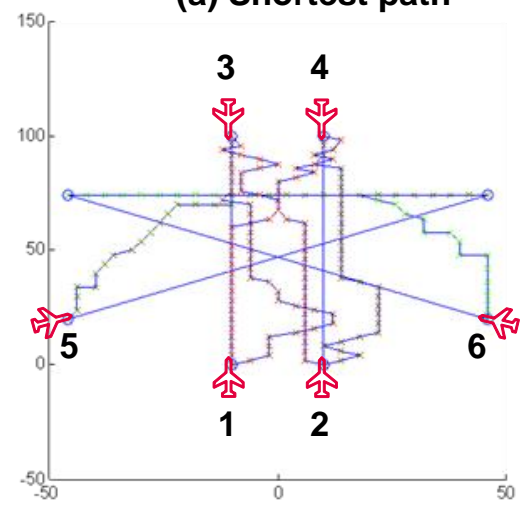

(b) Adaptability

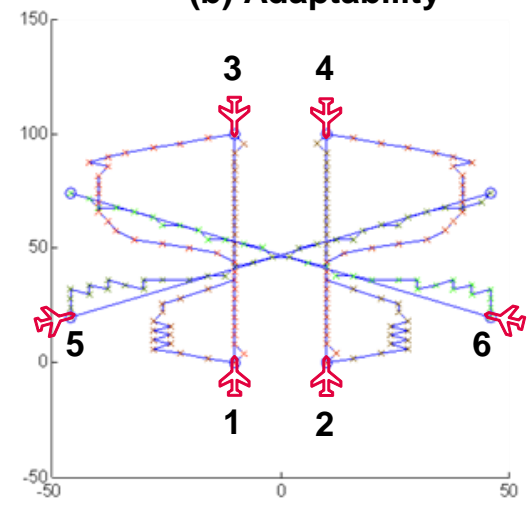

(c) Robustness

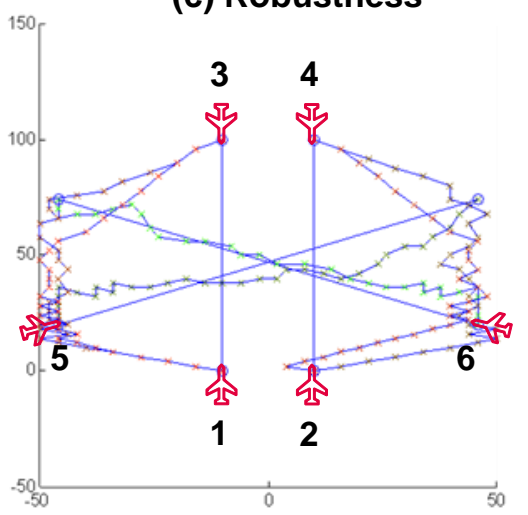

Figure 5. Example demonstrating analysis scenario

Figure 5 shows an example of one trajectory generated using each of the three cost functions (Equations 4 through 6 in figures 5.a through 5.c respectively). The ownship aircraft maneuver using path stretching and speed in order to meet the RTA while avoiding the intruder aircraft. (The speed profile is not shown in the figure.) The effect of the discrete time and heading is clear in the jagged nature of the trajectories. This analysis uses simplified trajectory representation as a series of discrete segments that are 2 minute long. While this representation is sufficient to test the hypothesis posed in this paper, in reality, the trajectories would be smoothed by introducing additional fidelity in the trajectory generation algorithm to reduce sharp heading changes for example.

Some observations can be made from Figure 5 on the path stretch decisions made by the aircraft when using shortest path (a), versus adaptability (b), versus robustness (c). When using shortest path (in Figure 5.a), the aircraft path stretch did not exhibit a clear pattern. When using adaptability (in Figure 5.b), the aircraft performed the path stretch in the early part of the trajectory, while it attempted to stay along the line of sight for the remainder of the trajectory. This is explained from Figure 3, where the adaptability metric has the highest value along the line of sight when there are no hazards or traffic. When there is traffic and other hazards, adaptability is higher where there are less of them. This is the reason why aircraft 1, 4, and 5 path-stretched to their left while aircraft 2, 3 and 6 pathstretched to their right. When using robustness (in Figure 5.c), aircraft 1, 2, 3, and 4 performed a path stretch to the extremity of the solution space. This is explained from Figure 4, where robustness has the highest values as far away from the traffic and hazards as possible. Aircraft 5 and 6 remained closer to their line of sight, where the extremities of the solution space are impacted by the other aircraft. Note that the robustness metric used in this paper is a special case that measures the probability of infeasibility due to the intruder aircraft but does not account for loss of feasibility due to other constraints such as the RTA and the heading and speed limits. It also measures this probability assuming a completely random state disturbance without accounting for constraint disturbances.

\section{F. Results and Observations}

Because of the random breaking of ties in the cost function, trajectories in addition to the one shown for demonstration in Figure 5 are required for inference. Figure 6 shows initial observations using ten runs of each of the cases introduced in Figure 5. All trajectories of each aircraft have the same duration, as they all meet the same RTA. All maneuvered trajectories are also conflict free from the five intruder aircraft original trajectories. However, a maneuver trajectory may lose separation with the other aircraft maneuver trajectory because of their simultaneous non-coordinated maneuvering. The only difference between the ten runs in each case is the random breaking of ties in the cost function. 
(a) Shortest path

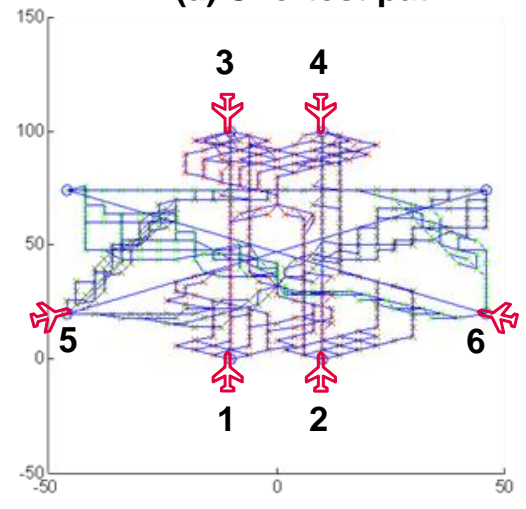

(b) Adaptability

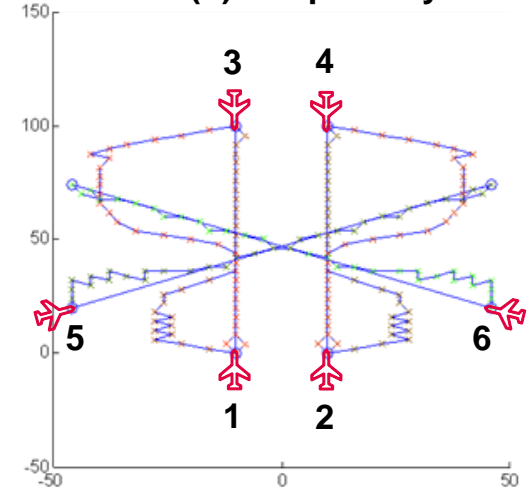

(c) Robustness

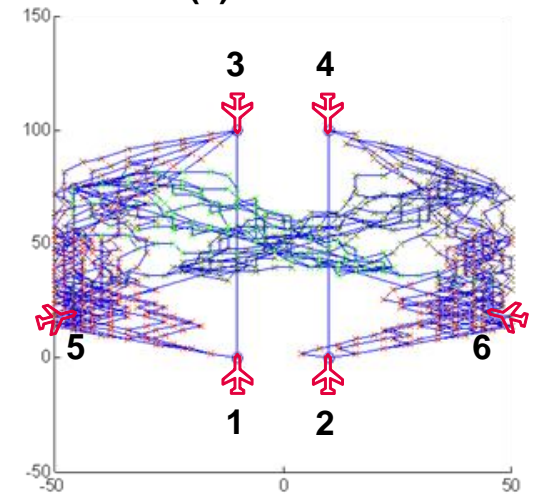

Figure 6. Initial observations demonstrating reduced variability using adaptability

Figure 6 demonstrates clearly the impact of using the adaptability and robustness metrics compared to the shortest path metric. Initial insights can be observed regarding the potential for secondary conflicts based on the amount of overlap between the trajectories. In case (a), using the shortest path reduces the variability in the trajectory path stretching. However, some randomness still exists, resulting in some trajectories infringing on each other. This infringement represents the potential for conflict between these trajectories.

Case (b) demonstrates that using adaptability resulted in a very consistent trajectory generation over the ten trials. (The plot in case b includes ten trajectories that are identical and mostly overlapping exactly.) The low variability in this case is due to the ability of the adaptability metric to finely differentiate between the available trajectories in the solution space based on their adaptability. Hence, fewer ties result, and the variability is much smaller. (Notice that there is a tie in the last time step near the destination which results in slight variability.) Adaptability is also highest along the line of sight between the aircraft current position and the destination (see Fig. 3). Therefore, the trajectory that is highest in adaptability tries to remain as close as possible to the line of sight. The compact nature of the adaptable trajectory and the low variability because of the low number of ties in turn result in much lower overlap between the maneuver trajectories. Therefore, using adaptability, which is a measure of trajectory flexibility, is able to reduce the potential for secondary conflicts in the process of maneuvering to resolve primary conflicts. The resulting solution is hence more stable, in the sense of needing fewer revisions.

In case (c), using robustness generated trajectories close to the borders, where they have least possibility to have conflict with other aircrafts. Using robustness kept a significant amount of variability in the trajectories generated. This result is due to a significant amount of ties in the cost function. An examination of the cost function indicated that, because of the limited amount of traffic in this scenario, the ratio of the number of feasible trajectories to the total number of trajectories is quite often equal to one (when all the available trajectories are feasible). These ties are broken randomly resulting in a significant amount of variability, and hence possible infringement between trajectories.

The number of secondary conflicts between the maneuvered trajectories of the six aircraft is then estimated according to the aircraft trajectories shown in Figure 6. One million $\left(10^{6}\right)$ possible combinations can be made using each of ten maneuvered trajectories for each aircraft. Because of the large time needed to estimate the secondary conflicts for all the one million combinations, only 15,625 combinations are picked randomly using five runs of each aircraft. Figure 7 shows the average, minimum, and maximum number of impending secondary conflicts in a 10minute horizon at each time step. Case (c) maximizing robustness and case (b) maximizing adaptability show less secondary conflicts than the shortest path case, and robustness shows the least creation of secondary conflicts. In the robustness case (c), the patterns of the trajectories, which stayed as far from the other traffic as possible, reduced the potential of secondary conflicts significantly, outweighing the effect of the randomness. The shortest path generated up to seven secondary conflicts during the whole duration, while robustness and adaptability generated only three secondary conflicts. The three aircraft pairs in conflicts for case (b) using adaptability are aircraft 1-5, 2-6 and 5-6. Because of the symmetric geometry of this scenario, the aircraft 1-5, 2-6 and 5-6 maneuvered symmetrically using adaptability. The secondary conflicts for case (c) using robustness are also found between aircraft 1-5, 2-6 and 5-6. That is because both aircraft 1 and 5 ( 2 and 6) went to the left (right) border when using robustness. The cumulative conflict time was also estimated for all three cases. Based on one tail paired $t$ test, the cumulative conflict time of 
shortest path case was also found to be statistically greater than the cases maximizing adaptability and maximizing robustness with probability of $99.9 \%$.

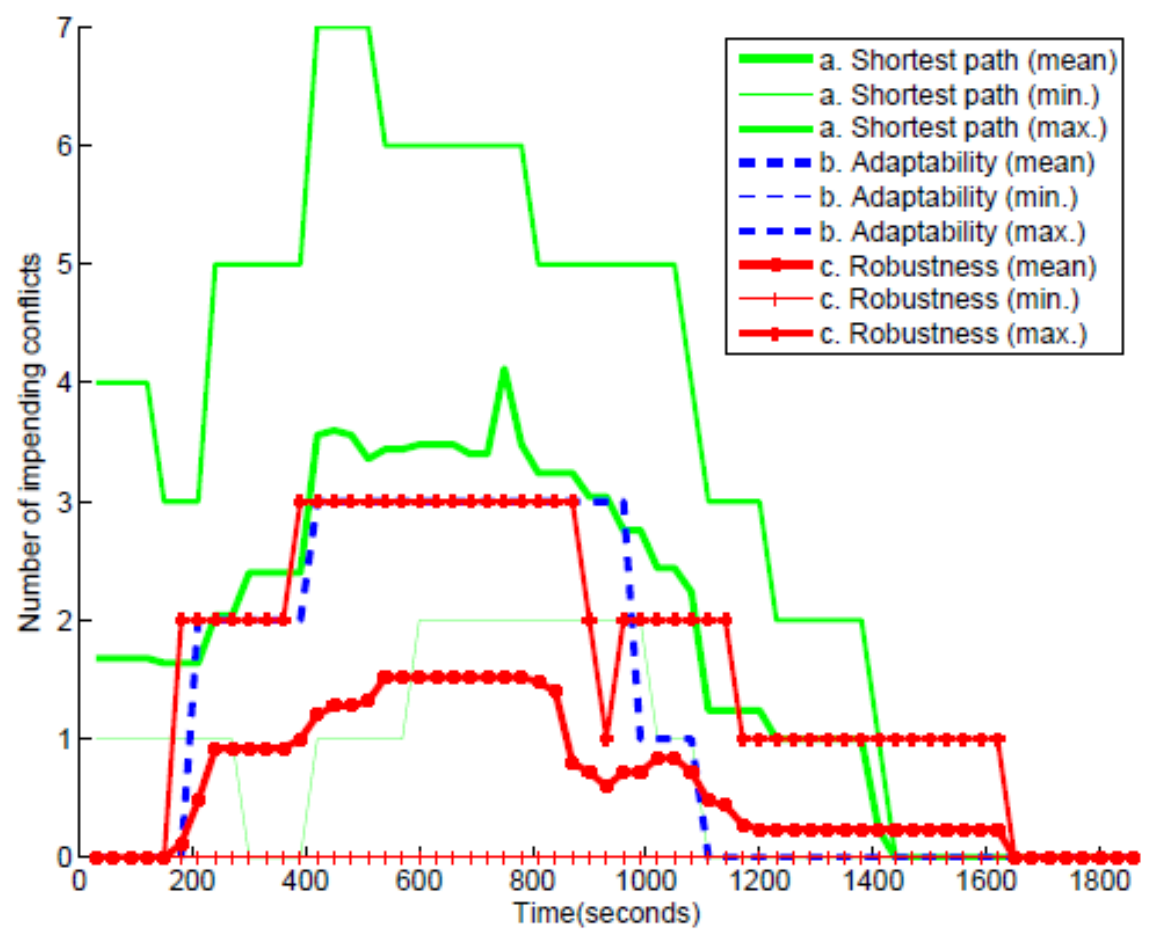

Figure 7. Observations demonstrating reduced secondary conflicts using robustness and adaptability

\section{Conclusions and Future Extensions}

The analysis reported in this paper demonstrated that using flexibility metrics in planning flexible aircraft trajectories results in improving the stability of separation assurance. The impact was quantified using the potential for secondary conflicts observed in hypothetical scenarios involving aircraft resolving conflicts with intruder aircraft without coordination. The scenario demonstrated that using trajectory flexibility, represented by the quantified metrics of trajectory robustness and adaptability, reduces the potential for secondary conflict between aircraft relative to using a shortest path criterion. These flexibility metrics can be combined with other metrics in the trajectory planning of pilots, airlines, and traffic managers. By incorporating these metrics, the stability of separation assurance can be improved, even without explicit coordination among aircraft or for the aircraft by a ground system.

\section{Acknowledgment}

This research was funded by NASA under contract NNA07BA86C.

\section{References}

${ }^{1}$ Joint Planning and Development Office, "Next Generation Air Transportation System Integrated Plan," URL: http://www.jpdo.gov/library/NGATS_v1_1204r.pdf.

${ }^{2}$ Wing, D., "A Potentially Useful Role for Airborne Separation in 4D-Trajectory ATM Operations," Proceedings of the $5^{\text {th }}$ AIAA Aviation Technology Integration and Operations (ATIO) Conference, AIAA-2005-7336, 2005.

${ }^{3}$ Green, S. M., Bilimoria, K. D., and Ballin, M. G., "Distributed Air/Ground Traffic Management for En Route Flight Operations." Air Traffic Control Quarterly, Vol. 9, No. 4, 2001, pp. 259-285.

${ }^{4}$ Idris, H., Vivona, R., Penny, S., Krozel, J., and Bilimoria, K., "Operational Concept for Collaborative Traffic Flow Management based on Field Observations," Proceedings of the $5^{\text {th }}$ AIAA 5th Aviation Technology, Integration and Operations (ATIO) Conference, AIAA-2005-7434, 2005. 
${ }^{5}$ Krishnamurthy, K., Barmore, B., and Bussink, F., "Airborne Precision Spacing in Merging terminal Arrival Routes," $6^{\text {th }}$ USA/Europe Air Traffic Management R\&D Seminar, 2005.

${ }^{6}$ Blom H.A.P., B. Klein Obbink, and G.I. Bakker, "Safety Risk Simulation of an Airborne Self Separation Concept of Operation," Proceedings of the $7^{\text {th }}$ AIAA Aviation Technology Integration and Operations (ATIO) Conference, AIAA 2007-7729.

${ }^{7}$ Barhydt, R., and Kopardekar, P., "Joint NASA Ames/Langley Experimental Evaluation of Integrated Air/Ground Operations for En Route Free maneuvering," $6^{\text {th }}$ USA/Europe Air Traffic Management R\&D Seminar, 2005.

${ }^{8}$ Mediterranean Free Flight Programme Final Report, D821, http://www.medff.it/public/index.asp, November 2005.

${ }^{9}$ Erzberger, H., T.J. Davis, and S.M. Green, “Design of Center-TRACON Automation System," AGARD Meeting on Machine Intelligence in ATM, Berlin, Germany, 1993.

${ }^{10}$ Idris H., D. Wing, R. Vivona, and J.L. Garcia-Chico, "A Distributed Trajectory-Oriented Approach to Managing Traffic Complexity," Proceedings of the $7^{\text {th }}$ AIAA Aviation Technology Integration and Operations (ATIO) Conference, AIAA-2007-7731, 2007.

${ }^{11}$ Idris H., R. Vivona, J.L. Garcia-Chico, and D. Wing, "Distributed Traffic Complexity Management by Preserving Trajectory Flexibility," Proceeding of the 26 $6^{\text {th }}$ Digital Avionics Systems Conference, 2007.

${ }^{12}$ Idris H, T. El-Wakil, and D. Wing, "Trajectory Planning by Preserving Flexibility: Metrics and Analysis," Proceedings of the AIAA Guidance Navigation and Control (GNC) Conference, AIAA-2008-7406, 2008.

${ }^{13}$ Idris H., and R. Vivona, "Metrics for Traffic Complexity Management in Self-Separation Operations," Air Traffic Control Quarterly, Volume 17, Number 1, 2009.

${ }^{14}$ Idris H., D. Delahaye, and D. Wing, "Distributed Traffic Flexibility Preservation for Traffic Complexity Mitigation ," $8^{\text {th }}$ USA/Europe Air Traffic Management R\&D Seminar, 2009.

${ }^{15}$ Idris H, N. Shen, T. El-Wakil, and D. Wing, "Analysis of Trajectory Flexibility Preservation on Traffic Complexity," Proceedings of the AIAA Guidance Navigation and Control (GNC) Conference, 2009. 